Archives

$24 \mid 2000$

La séduction

\title{
Portrait de jésus en séducteur
}

Jean-Pierre Albert

\section{(apenEdition \\ Journals}

\section{Édition électronique}

URL : http://journals.openedition.org/ccrh/1942

DOI : $10.4000 /$ ccrh. 1942

ISSN : $1760-7906$

\section{Éditeur}

Centre de recherches historiques - EHESS

Édition imprimée

Date de publication : 20 avril 2000

ISSN : 0990-9141

\section{Référence électronique}

Jean-Pierre Albert, «Portrait de jésus en séducteur », Les Cahiers du Centre de Recherches Historiques [En ligne], 24 | 2000, mis en ligne le 16 janvier 2009, consulté le 21 avril 2019. URL : http:// journals.openedition.org/ccrh/1942 ; DOI : 10.4000/ccrh.1942

Ce document a été généré automatiquement le 21 avril 2019

Article L.111-1 du Code de la propriété intellectuelle. 


\title{
Portrait de jésus en séducteur
}

\author{
Jean-Pierre Albert
}

1 Ce qui suit n'est peut-être rien de plus qu'un exercice d'école, dont la règle du jeu serait la suivante : construire, à propos d'un phénomène quelconque, une hypothèse improbable et la soutenir avec une rigueur (ou une obstination) suffisante pour voir jusqu'à quel point elle éclaire, par contraste ou par analogie, ce que nous croyons être la réalité. En l'occurrence, notre propos commun est d'esquisser une histoire de la séduction. Je propose de considérer que la vocation religieuse, et plus particulièrement la vocation des saintes mystiques, peut être analysée comme une forme de séduction exercée par Jésus sur des jeunes filles. Si cette hypothèse est totalement arbitraire, il n'en résultera pas grand-chose. J'ai quelques raisons de croire qu'elle ne l'est pas tout à fait, et que l'expérience mérite d'être tentée ${ }^{1}$.

2 Une histoire de la séduction doit restituer la manière dont cette réalité évolue dans le temps, et non simplement la place dans l'histoire d'une situation supposée plus ou moins identique à elle-même. Mais il faut bien partir d'un contenu de définition minimal pour identifier empiriquement des matériaux susceptibles d'alimenter le dossier. Une telle définition et ses éventuelles variations peuvent être recherchées au niveau sémantique, en examinant les emplois du terme, ses contextes, les situations auxquelles il fait référence. Or, dans le champ du religieux chrétien, il y a bien une place pour les notions de séduction ou de séducteur, mais elles se rapportent à l'œuvre du diable, jamais à Dieu. Pour les appliquer au personnage du Christ tel qu'il est mis en scène dans le discours des mystiques, il convient donc de dépasser le mot pour aller vers la chose, c'est-à-dire de comparer des descriptions d'expériences mystiques à des descriptions de relations amoureuses relevant de ce qu'une époque désigne comme des figures de la séduction. Cette enquête serait plus aisée si l'on disposait déjà d'une histoire de la séduction dans le domaine de l'amour profane. Mais puisque c'est précisément cette histoire qu'il s'agit d'esquisser, j'ai dû partir d'un modèle de la séduction sans référence historique précise, susceptible toutefois de devenir un objet pour l'histoire. L'hypothèse la plus vraisemblable est que ce modèle détermine largement le vécu de l'amour mystique. Il serait, toutefois, piquant d'imaginer que le contraire est également vrai. Faute de pouvoir 
trancher cette question, je me contenterai de présenter un parallèle entre les deux types de situations et d'en tirer quelques conséquences utiles à notre propos.

\section{Mariage et séduction}

3 La notion de séduction réfère à la fois à une réalité psychologique et à une réalité sociale. Comme expérience vécue d'une interaction entre deux personnes, elle désigne une attirance irrépressible pouvant aller jusqu'à une perte (momentanée ou plus durable) de sa liberté de jugement et d'action. En même temps, cette attirance est ressentie comme plus ou moins dangereuse, il se peut que l'on tombe sous la coupe d'un "séducteur ", d'une personne déployant sciemment une stratégie du faux-semblant ou du mensonge. Le risque d'illusion est d'autant plus grand que la relation de séduction est virtuellement réciproque: si l'on veut me séduire, c'est que j'ai moi-même séduit. Et cela reste valorisant, même si je reconnais l'artifice déployé par le séducteur. Une réputation « d'homme à femmes » n'a jamais nui aux Don Juan de tout poil, bien au contraire...

D'un point de vue social, et conformément à l'étymologie, la séduction écarte d'un « droit chemin ». Pour une jeune fille, jusqu'à une période récente, ce «droit chemin » était, en règle générale, celui d'un mariage approuvé par les parents. Céder à un séducteur, c'est prendre le risque de se retrouver bientôt "séduite et abandonnée ", et du même coup indisponible pour une union légitime. Ce n'est sans doute pas un hasard si, dans le Dom Juan de Molière, la principale scène où l'on voit le séducteur à l'œuvre le met en présence d'une jeune fille prête à se marier. La distance apparaît en effet maximale entre logique de la séduction et logique conjugale. On verra plus loin à quelles conditions il est possible que cette distance se réduise.

5 Les deux faces de la définition de la séduction se rejoignent ainsi dans l'idée d'une captation, ou d'une rupture avec l'ordre des conduites rationnelles et des attentes de normalité sociale. Cette rupture est inséparable de l'entrée en scène d'une logique du désir, ayant ses racines dans le corps. D'où le caractère perturbateur, transgressif, de la séduction, justifiant la réprobation sociale du séducteur et de sa victime; d'où également son association à l'image du diable, dans le double registre du mensonge et du désir charnel.

6 A première vue, il n'y a donc que peu de rapports entre la jeune fille qui se laisse séduire par un galant et celle qui se voue à Dieu. On peut cependant relever un isomorphisme entre les deux situations, dès lors que la vocation religieuse, tout comme un «coup de foudre ", devient un élément perturbateur au plan social. Or l'hagiographie est riche en récits de vocation ayant pour effet de remettre en cause un projet de mariage voulu par les parents. Somme toute, une défloration et un vœu de virginité également intempestifs ont pour effet commun d'invalider la perspective du mariage : Jésus, comme un séducteur mondain, capte une personne et la place hors du marché conjugal où sa famille voulait la confiner.

7 Le parallèle entre les deux situations est particulièrement sensible lorsque la vocation religieuse prend la forme d'une " conversion ", c'est-à-dire d'une rupture soudaine dans une existence qui semblait devoir se conformer à un schéma plus attendu. En fait, dans la vie des saintes, telle du moins qu'elle est reconstruite par les hagiographes ou les intéressées elles-mêmes, il n'y a guère de conversions «ex nihilo»: si la conversion au moment de l'adolescence reste un épisode à peu près inévitable, elle vient presque 
toujours confirmer une vocation précoce empêchée ou négligée. Même si ce dernier motif doit beaucoup aux conventions de l'hagiographie, on peut se demander s'il n'a pas un équivalent du côté de l'amour profane, avec la construction sociale d'une attente du "grand amour ", ou du « Prince charmant ", que la grâce d'une rencontre devra un jour ou l'autre satisfaire. Il conviendrait de dater ce système de représentations.

Quoi qu'il en soit, la proximité des deux registres de l'amour est parfois saisissante. Ainsi Sœur Faustine (Hélène Kowalska, 1905-1938), béatifiée depuis peu, a refusé de suivre sa vocation, manifestée dès l'âge de sept ans: "A dix-huit ans - écrit-elle dans son Journal -j'ai prié très instamment mes parents de me permettre d'entrer au couvent; ils repoussèrent catégoriquement ma demande. Après quoi, je me suis abandonnée aux vanités de la vie.» Un soir qu'elle est au bal avec une de ses sœurs (elle éprouve cependant des « tourments intérieurs »), Jésus lui fait une scène de jalousie : «Soudain, au moment où je commençais à danser, j'aperçus près de moi Jésus supplicié, dépouillé de ses vêtements, tout couvert de blessures, qui me dit ces mots : «Jusqu'à quand vais-Je te supporter et jusqu'à quand vas-tu Me décevoir ? " " Mais le ton change bien vite, et nous glissons à la description la plus convenue du coup de foudre: "À ce moment, la charmante musique cessa pour moi, la société où je me trouvais disparut à mes yeux, il ne restait que Jésus et moi. Je m'assis auprès de ma sœur, simulant un mal de tête pour cacher ce qui venait de se passer. » Hélène se rend ensuite à la cathédrale de la ville pour un véritable rendez-vous secret avec Jésus, qui lui dit : « Pars tout de suite pour Varsovie; là, tu entreras au couvent. » La suite nous ramène au schéma de la fuite avec le galant. Hélène charge sa sœur de prévenir ses parents de son départ : « Et ainsi, conclut-elle, avec une seule robe, sans bagages, j'arrivai à Varsovie. $»^{2}$

Jésus est donc venu « prélever » son amante dans le lieu même de la séduction mondaine, et a enfin obtenu le consentement qui lui était jusqu'alors refusé. De tels atermoiements n'ont rien d'exceptionnel. Comme dans les amours humaines, la conversion demeure marquée par un balancement entre le doute et le besoin d'une certitude subjective : le rapport qui se noue avec le Ciel est-il authentique? A cet égard, l'Église a développé des procédures d'expertises de plus en plus exigeantes qui, nourries aujourd'hui de psychologie ou de psychanalyse, n'en ont pas moins une fonction comparable à celle de la vieille démonologie. A l'échelle de la conscience individuelle, le besoin de certitude se satisfait principalement par la construction imaginaire de l'amour de l'autre : je n'ai pas à choisir parce que je suis choisie. La conversion - comme le « coup de foudre » - n'est ainsi que le reflet d'une élection aussi absolue qu'arbitraire, dont la réalité supposée se conforte souvent de la mise en scène d'une résistance du sujet. Témoin, ce dialogue entre Jésus et une mystique, Hélène Villefranche (1879-1951), extrait de son autobiographie :

- Seigneur, pourquoi m'avoir regardée... choisie... sans intelligence ni jugement ni, ce qui est pis, sans vertu d'aucune sorte?

- L'Amour ne justifie pas ses choix. Plus la misère est grande, plus elle fournit à la Miséricorde un piédestal glorieux.

- Oh, permettez-moi de vous le dire : vos fantaisies d'amour sont particulièrement inexplicables et votre miséricorde bien servie!

- Je t'ai choisie pour la réalisation d'un secret dessein d'amour...

- La réalisation d'un secret dessein !... J'ai peur... Où donc découvrirai-je le trou qui me permette d'échapper à votre poursuite ? J'y courrais afin d'y finir mes jours !... Et le psalmiste me répondait : « Où aller loin de ton esprit, où fuir loin de ta face? ». Alors, tremblante, mais vaincue par l'Amour, je finissais par me livrer ${ }^{3}$.

On pourrait souligner sans peine la charge érotique de ce pieux marivaudage, mais là ne me semble pas l'essentiel : comment dire l'amour mystique sans recourir au lexique de 
l'amour profane ? Il faut surtout noter que ce dialogue se situe à un moment où Hélène, qui a déjà refusé deux prétendants, hésite encore sur la manière dont elle accomplira sa vocation religieuse - dans le monde ou hors du monde -, son père ayant refusé qu'elle entre au couvent. En imaginant qu'elle résiste autant qu'elle le peut aux avances insistantes de Jésus, elle échappe à la pleine responsabilité de ce qui est, en réalité, son choix.

Dans ces deux exemples, Jésus joue donc, par rapport aux attentes des familles, un rôle perturbateur comparable à celui d'un séducteur ordinaire. Mais, loin d'emporter sa victime dans les délices de la chair, il la promet à l'ascétisme et à la souffrance. Cela ne suffit-il pas, en dépit des analogies déjà relevées, à éloigner décidément le colloque mystique du théâtre de la séduction? Il nous faut revenir, pour y voir plus clair, sur la place du corps dans cette expérience.

\section{Les aléas de la vie de couple}

12 Comme on l'a vu, l'intrusion de Jésus dans la vie d'une jeune fille joue un rôle analogue à celle d'un séducteur terrestre dans la mesure où elle exclut la forme socialement approuvée du mariage et ses conséquences souhaitées - au premier chef la naissance d'enfants légitimes. Le parallèle peut être poussé plus loin si l'on admet que les relations fondées sur la séduction sont considérées, traditionnellement, comme stériles ${ }^{4}$ : le corps érotique s'oppose au corps reproducteur comme les jeux du désir aux contraintes de l'institution conjugale. Et c'est bien, en effet, à ce corps érotique que doit renoncer la bonne épouse chrétienne, dont la chasteté est la réalisation imparfaite de l'idéal de virginité incarné par la plupart des « épouses du Christ ».

La vocation religieuse, lorsqu'elle débouche sur une vie rangée dans un couvent, offre donc une existence qui ressemble beaucoup plus à celle d'une épouse légitime qu'au parcours incertain d'une fille séduite : dans les deux cas, la femme jouit de la garantie d'une institution et est invitée à rompre avec toutes les intrusions perturbatrices d'une logique du désir, clairement marquée comme diabolique. En vérité, le couvent semble bien fait pour refroidir l'intensité émotionnelle des "conversions" les plus sincères. Jésus, d'abord séduisant, risque fort de se changer très vite en un mari austère, ou simplement absent. Or la carrière mystique semble, tout au contraire, parvenir à conserver intacte la force subversive du moment initial et sa richesse affective. Certes, le choix du Ciel impose de priver le corps de ce qui le rattache aux valeurs de la terre et, par conséquent, il requiert, à un premier niveau, la négation de la chair caractéristique de la vie consacrée - le plus souvent, du reste, les mystiques sont des religieuses. Mais elles doivent aussi lutter contre la routine desséchante de l'institution et, pour cela, elles imposent de manière subversive la réalité de leur corps. En d'autres termes, le corps d'une mystique se constitue dans la négation d'une première négation. La première est la négation-effacement d'un corps soumis à la régulation institutionnelle du mariage ou de la règle; la seconde, tout en préservant l'essentiel des effets de la première (rejet du plaisir physique et, lorsqu'il s'agit de religieuses, de la fécondité), se traduit par un « retour de la chair », par l'affirmation envahissante d'un corps souffrant et déréglé.

L'expérience des mystiques se présente ainsi comme la scène imaginaire de rapports personnels avec Jésus engageant le corps des deux partenaires. Cela suppose, entre autres choses, que le corps liturgique du Christ, l'hostie, redevienne en quelque façon un corps 
littéral, support d'une expérience à forte coloration affective. Nombre de mystiques sont saisies d'une boulimie eucharistique, vécue sous la forme littérale de l'appétit et du plaisir pris à l'ingestion de l'hostie, qui s'accompagne souvent d'une extase. L'hostie elle-même se refait chair, retrouve le goût du sang ou engendre toute une gamme d'images anthropophagiques. Outre le registre de l'alimentaire, la relation charnelle avec le Christ multiplie les figures d'un véritable corps à corps : transverbération, cœurs échangés ou arrachés, pénétration dans le cœur de Jésus par sa plaie au côté, etc. Le registre de la souffrance devient ainsi inséparable de celui de l'amour, et ce, d'autant plus qu'à ces scènes fantasmatiques correspondent souvent des lésions bien réelles du corps, volontaires ou involontaires. De façon générale, les prodiges dont le corps des saintes est le support sont autant d'expressions d'un absolu dérèglement, qui est supposé manifester l'emprise amoureuse d'un partenaire toujours avide de nouvelles preuves d'amour.

La relation mystique se situe ainsi dans le registre aventureux d'un engagement personnel et passionnel et non dans la sécurité d'un statut institué. Elle dessine, en cela, un modèle de "l'amour fou » qui reste marqué par l'intensité émotionnelle, mais aussi l'anomie du moment initial. La même chose existe du côté de l'amour profane : un roman comme Manon Lescaut illustre assez bien les errances et les douleurs d'une relation qui, fondée sur un " coup de foudre ", connaît tous les aléas liés à sa marginalité sociale et aux inévitables outrances de la passion. Les mystiques, quant à elles, projettent volontiers sur Jésus à la fois les désordres qui signifient leur distance par rapport à la règle commune et les crises de leur vocation. Cela se traduit par l'image d'un Christ tyrannique et inconstant, dont les intrusions intempestives dans la vie de ses amantes répètent la violence de la captation initiale. Ces désordres offrent la certification publique d'une aventure qui, sans cela, resterait purement intérieure, et contribuent à l'objectiver. Dans le cadre d'un couvent, il est ainsi fréquent que Jésus, comme sous l'emprise d'une passion irrépressible, vienne perturber les routines de la vie sous la règle. Pour ne citer qu'un seul exemple, la biographie de Josefa Menéndez (1890-1923), sœur de la Société du Sacré-Cœur de Jésus, comporte nombre d'épisodes tels que celui-ci :

Le samedi 26 novembre, vers trois heures de l'après-midi, Josefa travaille avec son ardeur habituelle aux uniformes des enfants, dans l'atelier du noviciat. Jésus l'y rejoint soudain.

" Je veux - dit-Il - que tu demandes à ta Mère la permission que Je reste un moment avec toi. »-Je partis aussitôt la demander, puis j'allai à la chapelle des CEuvres où il vint avec sa croix. ${ }^{5}$

Cette exigence de désordre se vérifie encore dans le cas, assez marginal, d'Anna-Maria Taïgi (1769-1837), une des rares bienheureuses officiellement reconnues ayant conjugué les manifestations les plus spectaculaires de la mystique à une vie d'épouse et de mère de famille. Jésus s'impose à elle peu après son mariage, et la meilleure volonté des hagiographes ne parvient pas à effacer le spectre de l'adultère des tableaux qu'ils nous décrivent. Est-ce un hasard si Jésus s'ingénie à perturber les activités ménagères de l'épouse, au grand dam du mari qui, écrit l'hagiographe,

[...] proche cousin du bonhomme Chrysale, a toujours attaché beaucoup plus d'importance à la bonne soupe de la sainte qu'à ses extases?

C'est que, entre l'action et la contemplation, le dilemme est constant :

Elle se mettait à nettoyer les casseroles, et soudain Notre-Seigneur était là. Elle s'empressait de détourner ses regards, puis, vaincue, demeurait ravie, sa casserole en main [...]. La Bienheureuse finit par se plaindre à Notre-Seigneur. « Laissez-moi tranquille, Seigneur. Retirez-vous ; j'ai mes occupations. Je suis mère de famille ! ${ }^{6}$. 
17 Mais, s'il lui arrive de se comporter en amant trop empressé, Jésus peut aussi abandonner durablement ses partenaires aux affres de la «sécheresse ». Dans ces moments de doute, elles sont parfois soumises aux pressions d'un autre séducteur, le Malin qui les agresse ou vient les tenter. Ces épisodes alternés d'heureuses extases et de solitude désespérée me semblent particulièrement révélateurs de l'incertitude essentielle d'une relation fondée sur le seul sentiment. En même temps, l'éventualité persistante d'une rencontre avec le Diable là où Dieu est attendu fait écho aux incertitudes des premiers temps de la vocation : justifiable lorsqu'elle est « divine ", la mystique porte toujours le soupçon du «diabolique», autre manière également crédible d'expliquer les inévitables transgressions qui la constituent. On retrouve ainsi à chaque étape l'ambivalence qui marque la notion même de séduction: il est toujours dangereux de sortir du «droit chemin » des institutions avec pour seule garantie une éblouissante certitude subjective. $\mathrm{Du}$ moins l'existence d'un espace légitime de la mystique «divine» offre-t-il une justification aux carrières religieuses déviantes. Pourrait-il en aller de même à l'égard des amours terrestres?

\section{Du « coup de foudre » au mariage d'amour}

Les exemples que j'ai cités sont empruntés à des biographies de mystiques des deux derniers siècles. En fait, il existe des cas analogues depuis, au moins, le XIV ${ }^{\mathrm{e}}$ siècle. Il est même possible de remonter plus haut encore si l'on cherche dans la littérature hagiographique non plus des exemples de captation mystique, mais des cas, symétriques et inverses, de " coups de foudre » masculins en présence de saintes que leurs amoureux fascinés tentent d'écarter de leur vocation spirituelle, jusqu'à les vouer au martyre lorsque leurs derniers espoirs sont déçus. Ces cas peuvent, à un premier niveau, s'expliquer dans le cadre de la condamnation chrétienne du désir charnel: ce sont toujours des païens qui cèdent au charme des belles vierges chrétiennes. Il n'en reste pas moins que les anciens hagiographes insistent toujours sur leur beauté, comme si celle-ci faisait partie des conditions de leur élection. La réciproque est également vraie. La beauté ineffable du Christ, «le plus beau des enfants des hommes", est un lieu commun de la mystique, au même titre que les descriptions effroyables de son corps supplicié. J'ai tenté ailleurs de montrer comment la beauté, d'abord gloire de la chair, peut aussi devenir un indice de spiritualité, à condition toutefois de perdre toute dimension érotique ${ }^{7}$.

Une même spiritualisation de la beauté corporelle et des amours humaines, pouvant aller jusqu'à une véritable mystique, existe dans notre tradition littéraire. Faute de dater ses origines, je crois pouvoir affirmer qu'elle est devenue, au cours des deux derniers siècles, une composante essentielle de notre définition de l'amour ${ }^{8}$. Cela permet peut-être de comprendre comment devient possible une réhabilitation des relations de séduction, du moins à travers la figure du "coup de foudre »; comment, également, le modèle du « mariage d'amour » a pu s'imposer contre une pratique de l'alliance étroitement soumise aux intérêts des familles. La toile de fond de ces mutations est bien évidemment la diffusion des valeurs individualistes, mais il n'est pas inutile de repérer les discours légitimants qui l'ont accompagnée.

Dans un texte célèbre de Madame Bovary - la scène des comices agricoles -, Flaubert met en scène avec toute la distance que l'on imagine le prototype de l'amour romantique et de 
sa justification. Le discours du conseiller vient d'exalter le devoir. Rodolphe, le séducteur, prend la balle au bond :

- Ah encore. Toujours le devoir. [...] Eh parbleu ! le devoir c'est de sentir ce qui est grand, de chérir ce qui est beau, et non pas d'accepter toutes les conventions de la société, avec les ignominies qu'elle nous impose.

- Cependant..., cependant..., objectait Madame Bovary.

- Eh non! pourquoi déclamer contre les passions? Ne sont-elles pas la seule belle chose qu'il y ait sur terre, la source de l'héroïsme, de l'enthousiasme, de la poésie, de la musique, des arts, de tout enfin?

- Mais il faut bien, dit Emma, suivre un peu l'opinion du monde et obéir à sa morale. - Ah ! c'est qu'il y en a deux, répliqua-t-il. La petite, la convenue, celle des hommes, celle qui varie sans cesse et qui braille si fort, s'agite en bas, terre à terre [...]. Mais l'autre, l'éternelle, elle est tout autour et au-dessus, comme le paysage qui nous environne et le ciel bleu qui nous éclaire.

21 Ce texte est sans nul doute caricatural. Mais le problème posé est bien celui de la légitimité d'un lien interpersonnel transgressif par rapport aux attentes de la société. Il ne devient acceptable (et même supérieur à la norme communément admise) que si la "passion » est posée comme un absolu, au même titre que l'amour divin des mystiques. Rodolphe invoque, en particulier, la dimension esthétique de la passion amoureuse et la situe dans le registre des valeurs nobles. Cette esthétisation de la vie suppose le dépassement de la condamnation du désir, réduit à l'ordre du corporel par la métaphysique dualiste: l'évidence du «coup de foudre» doit signifier bien plus que l'attirance sexuelle, elle résume un engagement, qui ne saurait mentir, de la personne tout entière. Cela suppose une redéfinition de la figure du séducteur qui, loin d'être un maitre en faux semblants, sera plutôt l'homme de l'authenticité, qui séduit par ce qu'il est vraiment en laissant deviner la profondeur» de sa personnalité. Passage du "beau parleur » au "beau ténébreux ». Le Christ des mystiques ne tient-il pas, lui aussi, son pouvoir de fascination de l'aura de mystère qui l'entoure?

Il semble donc que la séduction amoureuse ne puisse conquérir sa légitimité que par un renversement des valeurs qui lui étaient d'abord associées : de présomption de mensonge, elle doit devenir indice de vérité; d'abord fragile certitude subjective peu armée pour résister aux «bonnes raisons » du discours social, elle doit exprimer de façon synthétique une élection mutuelle, l'harmonie incompréhensible de deux cœurs. Aussi, loin de s'opposer au mariage, elle prépare son véritable accomplissement, le «mariage d'amour ». Ce retournement, mis en lumière par d'autres intervenants à cette journée, me semble un moment essentiel de l'histoire de la séduction. Mais est-il réellement datable, sinon au stade de sa quasi-généralisation? Ou bien n'est-il qu'un possible discours de l'amour, accessible à d'autres époques au moins de façon marginale?

Le miroir de l'amour mystique ne simplifie pas la réponse à ces questions. Peut-être permet-il de mettre au jour un modèle de comportement et de justification qui anticipe la reconnaissance d'une légitimité de la passion amoureuse socialement perturbatrice. On peut y lire en même temps des modalités de normalisation de ce lien passionnel, parallèles aux idéaux du mariage d'amour, dans la mesure où l'état de sainte mystique (vivante) devient un statut socialement reconnu, même s'il ne débouche sur aucune véritable institutionnalisation. La scène fantasmatique de l'amour mystique, justement parce qu'elle est aussi irréelle (et aussi réelle) que celle de la littérature, ne pourrait-elle pas être considérée comme un des laboratoires où s'est inventé « l'amour en Occident »? Ou bien n'est-elle que le reflet d'une histoire de la subjectivité qui se jouait ailleurs ? Dans les deux hypothèses, il reste vrai que cette nouvelle expérience du sujet a trouvé dans 
l'écriture mystique une expression abondante et précoce et que, en inventant des formes très subtiles d'attention à soi-même, elle a pu contribuer à l'éducation sentimentale de nos sociétés.

\section{NOTES}

1. Le dossier présenté ici s'appuie sur mon livre Le Sang et le Ciel. Les saintes mystiques dans le monde chrétien ; Paris, Aubier, 1997, 458 p., et plus particulièrement sur son chap. IV.

2. Petit journal de Sœur Faustine, Marquain, Ed. Jules Hovine, 1985, 37-38.

3. Père Emile Dubois, Une mission prophétique. Hélène Villefranche, 1879-1951, Montsûr, Éd. Résiac, 1976, p. 31.

4. Yvonne Verdier, Façons de dire, façons de faire, Paris, Gallimard, 1979, $370 \mathrm{p}$.

5. Josefa Menéndez, Un appel à l'amour, nouvelle édition, Toulouse, Éditions de l'Apostolat de la prière, 1972, p. 193.

6. Albert Bessieres, S. J., La Bienheureuse Anna-Maria Taïgi, mère de famille, Paris, Desclée de Brouwer, 1936, p. 134-137.

7. Jean-Pierre Albert, «Les belles du Seigneur », Communications, $n^{\circ} 60,1995$, p. 63-74.

8. Sur les liens entre érotique et mystique, je songe en particulier à la poésie de Baudelaire, à ce vers, par exemple, évoquant le corps de l'aimée : «Sa chair spirituelle a le parfum des anges ». On notera également que la psychanalyse, loin de démystifier ces "affinités électives", a été impliquée par André Breton dans une théorie de "l'amour fou » qui laisse toute sa place au mystère et à la magie de la rencontre amoureuse. 